\title{
SEND Version 3.0 Draft B
}

National Cancer Institute

\section{Source}

National Cancer Institute. SEND Version 3.0 Draft B. NCI Thesaurus. Code C90454.

The B draft of the standard for the exchange of non-clinical data (SEND) implementation guide. 